\title{
Metabolic syndrome biomarkers and prostate cancer risk in the UK Biobank
}

Maria J Monroy-Iglesias ${ }^{1}$; Beth Russell ${ }^{1}$, PhD; Danielle Crawley ${ }^{1}, \mathrm{PhD}$; Naomi E Allen ${ }^{2}$, PhD; Ruth C Travis $^{3}$, PhD; Aurora Perez-Cornago ${ }^{3}$, PhD; Mieke Van Hemelrijck ${ }^{1^{*}}$, PhD; Kerri Beckmann ${ }^{{ }^{*}}$, $\mathrm{PhD}$

*Both senior authors contributed equally

\section{Affiliations}

1) Translational Oncology and Urology Research (TOUR), School of Cancer and Pharmaceutical Sciences, King's College London, London, UK.

2) Clinical Trial Service Unit and Epidemiological Studies Unit, Nuffield Department of Population Health, Big Data Institute, University of Oxford, UK.

3) Cancer Epidemiology Unit, Nuffield Department of Population Health, University of Oxford, UK.

Corresponding author/address: Kerri Beckmann, Translational Oncology and Urology Research (TOUR), School of Cancer and Pharmaceutical Sciences, King's College London, London, UK. Email: kerri.beckmann@kcl.ac.uk

Word count: 250 (abstract); 3,763 (manuscript)

Key words: prostate cancer, metabolic syndrome, glycated haemoglobin, mediation analysis. 
Abbreviations: BMI: body mass index; BP: blood pressure; CDE: controlled direct effect; CFT: calculated free testosterone; $\mathrm{Cl}$ : confidence interval; CRP: C-reactive protein; CVD: cardiovascular disease; DAG: directed acyclic graph; HbA1c: glycated haemoglobin; HDL: HDLcholesterol; HR: hazard ratio; ICD-10: international classification of diseases tenth revision; IGF1: insulin-like growth factor 1; IGFBP: insulin-like growth factor binding protein; IGR: impaired glucose regulation; INTmed: mediated interaction; INTref: reference interaction; MET: metabolic equivalent of task; MetS: metabolic syndrome; NCEP ATP III: American diabetes association and national cholesterol education program III; NHS: national health service, NHSCR: national health service central register; OR: odds ratio; PCa: prostate cancer; PIE: pure indirect effect; PSA: prostate-specific antigen; RR: relative risk; SES: socioeconomic status; SHBG: sex-hormone binding globulin; T2DM: type 2 diabetes mellitus; TG: triglycerides; TT: total testosterone; WC: waist circumference; WCRF: ; WHO/IDF: world health organisation/international diabetes federation.

\section{Article category: Cancer Epidemiology}

\section{Novelty and Impact:}

Evidence for the association between metabolic syndrome (MetS), and its components, and prostate cancer (PCa) remains elusive. In this large, prospective study, we found that MetS is not associated to PCa risk. However, glycated haemoglobin ( $\mathrm{HbA1c}$ ) is inversely associated with PCa. When performing mediation analysis to examine potential hormonal and inflammatory 
pathways, total testosterone (TT), C-reactive protein (CRP) and insulin-like growth factor 1 (IGF-1) were identified as potential mediating factors for the association between $\mathrm{HbA} 1 \mathrm{c}$ and PCa. 
Abstract 250 (Max.) Adapted for Int J Can

We investigated the association between metabolic syndrome (MetS) and its components and risk of prostate cancer (PCa) in a cohort of men enrolled in the UK Biobank.

Our study cohort included 220,622 PCa-free men with baseline measurements of triglycerides (TG), HDL-cholesterol (HDL), glycated haemoglobin (HbA1c), blood pressure (BP), and waist circumference (WC). Multivariable Cox proportional hazards regression was used to analyse associations with PCa for: individual metabolic components (TG, HDL, HbA1c, BP, WC), combinations of two and three components, and MetS overall (three or more components). We conducted mediation analyses to examine potential hormonal and inflammatory pathways (total testosterone (TT), C-reactive protein (CRP), insulin-like growth factor 1 (IGF-1)) through which MetS components may influence PCa risk.

A total of 5,409 men in the study developed PCa during a median follow-up of 6.9 years. We found no significant association between MetS and PCa risk (Hazard Ratio (HR): 0.99, 95\% Confidence Interval (Cl): 0.92-1.06). No associations were found with PCa risk and individual measurements of TG, HDL, BP or WC. However, an inverse association was observed with elevated $\mathrm{HbA} 1 \mathrm{c}$ ( $\geq 42 \mathrm{mmol} / \mathrm{mol}$ ) (HR: $0.89,95 \% \mathrm{Cl}: 0.79-0.98)$. Consistent inverse associations were observed between $\mathrm{HbA} 1 \mathrm{c}$ and risk of PCa. Mediation analysis revealed TT, CRP and IGF-1 as potential mediating factors for this association contributing $10.2 \%, 7.1 \%$ and $7.9 \%$ to the total effect, respectively. 
Overall MetS had no association with PCa risk. However, a consistent inverse association with PCa risk was found for HbA1c. This association may be explained in part through hormonal and inflammatory pathways.

\section{Introduction}

Metabolic syndrome (MetS), which refers to a collection of metabolic risk factors for cardiovascular disease (CVD) and type 2 diabetes mellitus (T2DM), is a major cause of morbidity and mortality worldwide (1). Depending on the definition used, with the American Diabetes Association and National Cholesterol Education Program (NCEP ATP III) definition being the most commonly used, MetS includes at least 3 of the following: central obesity, increased serum triglyceride (TG) levels, decreased serum HDL cholesterol (HDL) levels, raised blood pressure (BP), and impaired glucose regulation (IGR), including prediabetic and diabetic glycaemic levels (2).

There is growing evidence suggesting that metabolic syndrome (MetS) may play a role in the development and/or diagnosis of PCa (3). However, whether the risk of PCa is increased or decreased and by what mechanism remains unclear. Several studies have focused on the individual factors of MetS, but few have studied all 5 components combined (4-7).

A number of studies have linked obesity to PCa. In the World Cancer Research Fund International (WCRF) 2014 report, body mass index (BMI) was positively associated with the risk of aggressive PCa (Relative risk (RR): 1.08, 95\% confidence interval (Cl): 1.04-1.12, per 5 $\mathrm{kg} / \mathrm{m}^{2}$ ), but no association was found with overall incidence of PCa (8). Waist circumference (WC) which is often used as proxy for abdominal (or central) obesity, has been linked to a decreased risk of overall PCa $(6,9)$, but an increased risk of advanced disease (8). 
Elevated glucose levels, including pre-diabetic and diabetic levels, have been consistently shown to be associated with reduced PCa risk (10). However, there is evidence linking T2DM with increased risk of high-grade PCa (Gleason score 8-10, or clinical stage >T3 or N1 or M1) (11).

Several underlying biological mechanisms have been suggested for the association between MetS and risk of overall and aggressive Pca. These mechanisms include increased serum levels of insulin-like growth factor 1 (IGF-1) (12) and chronic inflammation - which are both commonly observed in patients with MetS and may lead to an uncontrolled proliferative response and PCa carcinogenesis $(13,14)$. Conversely, MetS or its components, may lead to modifications of sex steroid pathways resulting in increased sex hormone binding globulin (SHBG), and decreased free and total testosterone levels which could lower PCa risk (15). Additionally, some studies have proposed that the overall risk of PCa may be influenced by lower prostate-specific antigen (PSA) levels, often seen in MetS and its individual components, which mask the presence of PCa or delay its detection, leading to an apparent reduced risk (16). The current study aimed to investigate the association between MetS components, both separately and combined, with overall PCa risk in the prospective UK Biobank. We also aimed to examine possible biological pathways through which the components identified may be affecting PCa risk by conducting mediation analyses.

\section{Materials and methods}

\section{Study population}


Our study population consisted of participants enrolled in the UK Biobank, a prospective cohort of over 500,000 men and women aged 40 to 69 years, registered with the UK National Health Service (NHS) who were recruited between 2006 and 2010 ( $n=229,131$ men). Participants were invited to attend one of the 22 assessment centres across England, Wales, and Scotland, where they were interviewed and underwent extensive physical examinations, and had biological samples collected $(17,18)$.

Self-reported data were collected at baseline from participants, using touch-screen questionnaires, and verbal interviews with a trained nurse (including information on sociodemographic factors, lifestyle characteristics, health-related factors, and prostate specific factors). Baseline assessment also included anthropometric measures (BMI, WC), and collection of biological samples (blood, urine and saliva). A more detailed description of the UK Biobank cohort can be found elsewhere $(17,18)$.

Eligible participants in the current study were men aged 40 to 69 years at assessment who had no record of prior $\mathrm{PCa}$ in national cancer registries. Men with no serum biomarker data available were also excluded. Men diagnosed with PCa within the first year of assessment were also excluded from the study cohort to reduce the possibility that findings were influenced by reverse causation, resulting in a final study sample of 220,622 men (Supplementary Figure 1).

Data on history of any other prior cancer diagnosis (ever, never) was determined through linkage with NHS Digital for participants residing in England and Wales, and the NHS Central Register (NHSCR) for participants residing in Scotland.

\section{Metabolic syndrome assessment}


The main exposure variables considered into our analyses included each of the MetS indicators. For these analyses, MetS components were categorized as continuous, quartiles and dichotomized variables with clinical cut-offs in accordance with the NCEP ATP III guidelines, as these are the most commonly used guideline to define MetS (see Supplementary Table 1) (19) (TG: $\geq 1.7 \mathrm{mmol} / \mathrm{L}, \mathrm{HDL}: \leq 1.00 \mathrm{mmol} / \mathrm{L}, \mathrm{WC}: \geq 102 \mathrm{~cm}, \mathrm{BP}: \geq 130 \mathrm{mmHg}$ (systolic) and/or $\geq 85$ $\mathrm{mmHg}$ (diastolic)). Since the percentage of participants with fasting glucose levels was low (4.3\%), we used the more stable measure of $\mathrm{HbA1C}$, based on the World Health Organisation/ International Diabetes Federation (WHO/IDF) guidelines for use of glycated haemoglobin (HbA1c) (20), with a clinical cut-off of $\geq 42.0 \mathrm{mmol} / \mathrm{mol}$ for the IGR criteria in our analyses. We defined overall MetS as the presence of any combination of three or more of the previously mentioned criteria. HbA1c was measured via high-performance liquid chromatography analysis on a Bio-Rad VARIANT II Turbo; HDL-cholesterol was measured by enzyme inmunoinhibition analysis on a Backman Coulter AU5800; Triglyceride levels were measured by GPO-POD analysis on a Beckman Coulter AU5800. Additional details about assay methods and quality control procedures are available online.

Potential mediating factors considered in secondary analyses included baseline serum concentrations of total testosterone (TT), calculated free testosterone (CFT), sex hormonebinding globulin (SHBG), C-reactive protein (CRP), and IGF-1 (21). CFT levels were calculated based on $T$, albumin and SHBG concentration according to the method described by Vermeulen et al (22), as follows: )/2N., where . Each of these factors was dichotomized as low or high concentrations according to defined clinical cut-off points as follows: low TT $\leq 8 \mathrm{nmol} / \mathrm{L}$, 
low CFT $\leq 0.255 \mathrm{nmol} / \mathrm{L}$, low SHBG $\leq 13 \mathrm{nmol} / \mathrm{L}$, high IGF-1 $\leq 10 \mathrm{nmol} / \mathrm{L}$, and high CRP $\geq 10 \mathrm{mg} / \mathrm{L}$ (23-25).

Date of diagnosis of all incident PCa [International Classification of Diseases Tenth revision (ICD10)=C61] up until December 31, 2016 and any previous cancer diagnoses. Data were not available for either stage or grade of disease.

\section{Data analyses}

Cox proportional hazards models were used to calculate hazard ratios (HRs) and $95 \% \mathrm{Cl}$ for PCa risk. Follow-up time was defined from one year after cohort entry until the date of PCa diagnosis, the date of death or end of follow-up (December 31, 2016), whichever came first.

The following covariates (assessed at baseline) were included as confounders: Age at entry in the UK Biobank cohort (40-49, 50-59, 60-69 years), ethnicity (Caucasian, Black, Asian, and mixed and other); socioeconomic status (SES, Townsend Deprivation Score, from 1 (most affluent) to 5 (most deprived))(26), physical activity (Metabolic Equivalent of Task (METs) per week (>10, 10$50,>50)$ based on self-reported walking, moderate and vigorous activity minutes per week)(27), BMI, self-reported history of PSA testing (never tested, ever tested or not known/missing), father diagnosed with PCa (no, yes, unknown), self-reported use of metformin (no, yes), selfreported use of statins (no, yes), and smoking status (never, former, current). Guided by directed acyclic graphs (DAG) to assess relationships between potential confounders (Supplementary Figure 2), we developed both a minimally adjusted model and a fully adjusted model to examine relationships between MetS (and its components) and PCa risk. The minimally adjusted model included: age at assessment, father with PCa, SES, and prior history 
of PSA testing. The fully adjusted model was further adjusted for ethnicity, history of other cancers, self-reported use of metformin, self-reported use of statins, BMI, physical activity, and smoking status.

Each of the MetS components, was considered both individually and with simultaneous adjustment for the other biomarkers. In order to determine which of the specific MetS components, or combinations, had greatest impact on PCa risk we conducted further analyses with different combinations of MetS components in separate models. These analyses were undertaken, with both minimal and full adjustment.

We also performed a series of sensitivity analyses to assess the influence of reverse causation and exclude associations potentially due to other cancers in relation to overall MetS and its individual components. These included: 1) considering all PCa diagnoses after assessment, including during the first year, 2) excluding PCa diagnoses within the first three years of followup and 3) excluding men with another cancer diagnosis prior to entering the UK Biobank Cohort.

\section{Secondary Mediation Analyses}

Due to consistent findings in our current study of an inverse association between levels of $\mathrm{HbA} 1 \mathrm{C}$ and risk of PCa, we conducted further analyses to examine potential biological pathways that might explain this apparent protective effect. Firstly, we examined variations in the concentrations of each potential mediating factor (TT, CFT, SHBG, CRP, and IGF-1) according to $\mathrm{HbA1c}$ levels in the total cohort using multivariable ordinary least squares regression to calculate geometric mean concentrations for each of these biomarkers. These analyses were 
made with a minimally adjusted model including: age, history of other cancers, ethnicity, and SES; and a model additionally adjusted for father with PCa, prior history of PSA testing, smoking status, use of metformin, use of statins, WC (quartiles), HDL (quartiles), and TG (quartiles), physical activity, and BMI. We then conducted mediation analysis to determine the percentage of the total effect that may be explained by each potential mediating factor adjusting for age, history of other cancers, ethnicity, SES, history of father with PCa, prior history of PSA testing, smoking status, use of metformin, use of statins, WC (low, high), HDL (low, high), and TG (low, high). We performed these analyses using the "med4way" command in STATA (28).

These analyses were allowed for the contribution of mediators including exposure-mediator interactions to be assessed (29). This method decomposes the total effect (in this case between HbA1c and PCa risk) into four components: controlled direct effect (CDE, the effect neither due to the mediator nor to exposure- mediator interaction), reference interaction (INTref: the effect only due to interaction), mediated interaction (INTmed: the effect due to interaction only active when mediation is present) and the pure indirect effect (PIE: the effect due to mediation alone), as explained in Supplementary Figure 3. The decomposition can be explained by the following equation (29): . We fitted a Cox proportional hazards regression model for the outcome (risk of PCa), and a logistic regression model for the mediators.

Analyses were performed using Stata version 15 (Stata Corporation, College Station, TX, USA). All statistical tests were two sided, with $p$ values $<0.05$ considered to be statistically significant. Proportional hazard assumptions were tested via Schoenfeld residuals and found to be satisfied. 


\section{Results}

A total of 5,409 men in the study developed PCa during a median follow-up time of 6.9 years. Participant characteristics are shown in Table 1. Median levels of each of the metabolic markers (TG, HDL, HbA1c, WC, and BP) among men who did and did not develop MetS are shown in Table 2.

Results of multivariable Cox proportional hazards regression examining the associations between MetS, and its various components, and risk of PCa are shown in Table 3. Overall MetS showed an inverse association in the minimally adjusted model (HR: $0.89,95 \% \mathrm{Cl}: 0.84-0.95)$ which disappeared in the fully adjusted model (HR: 0.99, 95\% Cl: 0.92-1.06). Further examination of each of the individual MetS indicators did not reveal associations between any individual components and PCa risk, with the exception of $\mathrm{HbA} 1 \mathrm{c}$, where a statistically significant inverse association was found (HR: $0.87,95 \% \mathrm{Cl}: 0.79-0.97)$. Additionally, associations between MetS individual components, as both continuous variables and categorized as quartiles, and PCa are shown in Supplementary Table 2. Similarly, these findings remained consistent in sensitivity analyses as shown in Supplementary Table 3.

Results of further analyses examining combinations of two and three specific MetS components and risk of PCa are also shown in Table 3. The strongest associations were seen for combinations that included $\mathrm{HbA} 1 \mathrm{C}$. For example, the combination of abnormal values of $\mathrm{HbA} 1 \mathrm{c}$ and $\mathrm{HDL}$ resulted in an $\mathrm{HR}$ of $0.73(95 \% \mathrm{Cl}: 0.59-0.91)$ and the combination of abnormal HbA1C and TG in an HR of $0.82(95 \% \mathrm{Cl}: 0.71-0.96)$ as compared to men with normal values of each of these components. 
For the three-component combinations, the three strongest associations were observed for combinations of abnormal values of HbA1c-HDL-WC (HR: $0.77,95 \% \mathrm{Cl}$ : 0.57-1.02); HbA1c-HDLTG (HR: 0.74, 95\%Cl: 0.58-0.96); and HbA1c-HDL-BP (HR: 0.69, 95\%Cl: 0.53-0.91).

\section{Potential mediators of HbA1c-PCa associations}

Serum levels for hypothesized mediating factors (expressed as adjusted geometric mean concentrations) are shown in Table 4 , according to whether men had high ( $\geq 42.0 \mathrm{mmol} / \mathrm{mol})$ or normal HbA1c levels. After adjusting for age, ethnicity, and SES, we found lower concentrations of TT, CFT, SHBG and IGF-1, and higher concentrations of CRP, in men with high HbA1c compared to men with low HbA1c (Model 1). The mean difference between biomarker levels was attenuated but remained after further adjustments (Model 2).

Results of mediation analyses are shown in Supplementary Table 4 and summarized in Figure 1. These analyses identified TT, IGF-1 and CRP as potential mediators of the association between impaired glucose regulation (I.e. high levels of $\mathrm{HbA1c}$ ) and PCa risk, however, with varying effects on the direction of the causal pathway. For example, participants with an increase in HbA1c were associated with lower odds of having increased levels of TT and IGF-1 (odds ratio (OR): $0.61,95 \% \mathrm{Cl}: 0.59-0.64$ and OR: $0.38,95 \% \mathrm{Cl}: 0.34-0.43$, respectively) and the proportion of the total effect mediated by these factors were $10.2 \%$ and $7.7 \%$, respectively. This means that overall, these factors caused a decrease in the overall PCa risk compared to the effect when these mediators were not considered. Conversely, patients with higher HbA1c levels were at increased odds of having higher CRP levels (OR: $1.87,95 \% \mathrm{Cl}: 0.99-1.25$ ) and this mediated the total effect by $7.1 \%$, thus increasing the overall PCa risk. 


\section{Discussion}

In our prospective study of 220,622 men, we found no significant association between MetS and risk of PCa. We also found no associations with WC, HDL, TG and BP, as individual components and PCa risk. However, there was an inverse association between HbA1c and PCa risk, which was also consistently seen in combinations with one or two other MetS components. Mediation analyses to identify potential pathways that might explain the inverse association between $\mathrm{HbA1c}$ and PCa risk, identified TT, IGF-1 and CRP as potential mediators.

Results of prior studies of overall MetS and PCa risk have been inconsistent. A recent metaanalysis by Gacci et al (13), which included 24 prospective studies that assessed the role of MetS in PCa risk and aggressiveness, found a positive association between MetS and overall PCa (Odds ratio (OR): 1.17, 95\% Cl: 1.00-1.36) and between MetS and high grade PCa (OR: 1.89, 95\% Cl: 1.50-2.38). Another meta-analysis reported a similar slight increase in overall PCa risk (10). On the other hand, our findings are in line with two cohort studies which reported no association between MetS and risk of total, advanced and high-grade PCa $(9,30)$.

Despite most studies showing null or weak positive associations between elevated glucose levels (including T2DM) and risk of any cancer (31), in the context of overall PCa risk most studies have reported an inverse association in the context of overall PCa (32). A recent systematic review of 15 prospective studies showed an inverse association between elevated glucose levels and PCa risk (pooled relative risk (RR) of $0.88,95 \% \mathrm{Cl}: 0.78-0.98)$ (33), while another meta-analysis of 19 prospective studies found an inverse association between T2DM and PCa (RR: $0.84,95 \% \mathrm{Cl}$ : 0.76-0.93)(34). Our findings support of an inverse association 
between impaired glucose regulation, assessed by $\mathrm{HbA1c}$ levels, and risk of PCa are inconsistent with most epidemiological studies.

While we found a decreased risk of overall PCa among men with some combinations including $\mathrm{HbA1c}$ and $\mathrm{HDL}$, low serum levels of $\mathrm{HDL}$ assessed as an individual risk factor were not associated with PCa risk. Several epidemiological studies suggest that dyslipidaemia is involved in the development of PCa (35). In a prospective cohort study of 69,735, Van Hemelrijck et al. (36) found that HDL was inversely associated with PCa risk (HR 0.79, 95\%Cl: 0.68-0.92), when comparing the highest with the lowest quartile. Mondul et al. (37) also found that men with higher HDL had a decreased risk of overall and advanced PCa. Hence, our finding of lower PCa risk in men with low HDL combined with high HbA1c is somewhat inconsistent with these previous reports. On the other hand, our finding of no association between TG and PCa risk, is in line with other studies (38).

Central obesity, has been independently associated with a decreased risk of overall PCa and an increased risk of advanced disease $(6,8,9)$. However, we found no associations between WC (the measure of central obesity most commonly used to define MetS) and risk of PCa.

The most commonly hypothesised pathways by which impaired glucose regulation (including T2DM) may affect PCa risk or aggressiveness, are through influences on sex steroid hormone levels, inflammatory pathways, or effects on growth factors (e.g.IGF-1). Low total testosterone, free testosterone and sex hormone-binding globulin serum levels are frequently seen in men with impaired glucose level (39).Therefore, low levels of sex steroid hormones could be contributing to the inverse association between hyperglycemia and PCa risk (40). Our mediation 
analysis, however, suggests that only total testosterone mediates the association between impaired glucose regulation (HbA1c levels) and PCa (explaining 10.2\% of the total effect).

IGF-1 is a peptide involved in the regulation of cell proliferation, differentiation and apoptosis. Several studies, including two meta-analyses, have found high concentrations of IGF-1 were associated with an increased risk of PCa $(41,42)$. IGF-1's bioavailability is regulated by insulinlike growth factor binding proteins (IGFBPs) and circulating levels of insulin (43). Insulin increases IGF-1 synthesis and decreases levels of IGFBP-1 and -2, and as a result, bioavailability of IGF-1 is increased. Therefore, hypoinsulinaemia, which may develop over time in diabetic patients, would have the inverse effect, reducing the bioavailability of IGF-1. In addition, there are studies that have shown that diabetic patients have lower levels of IGF-1. Results from our mediation analysis provide some support for the hypothesis that IGF-1 mediates the association between high levels of $\mathrm{HbA} 1 \mathrm{c}$ and PCa risk (explaining $7.9 \%$ of the total effect) by decreasing PCa risk.

However, it should be noted that our analyses included patients with both prediabetic and diabetic HbA1c levels and insulin levels were not available for this study.

In addition, accumulating data support the hypothesis that chronic inflammation contributes to prostate carcinogenesis through oxidative stress, DNA damage and altered gene expression (14). However, several recent studies have found no association between markers of inflammation (including CRP) and PCa. Unlike the other mediating factors investigated, CRP showed a positive association between high levels of $\mathrm{HbA1C}$ and $\mathrm{PCa}$, therefore implying that CRP would influence the direction of the total effect towards an increased PCa risk. This is consistent with previous studies that have found a clear association between insulin resistance 
and the chronic activation of pro-inflammatory signaling pathways (44). However, the net effect of HbA1c and PCa risk is negative when accounting for all the proposed mediating factors studied here. It should be noted that since the mediators tested in this paper only account for a small proportion of the total effect, there are likely to be other unidentified factor or pathways through which high HbA1c levels affect PCa risk.

In addition to the above proposed biological mechanisms, it has also been hypothesised that detection bias due to lower serum PSA levels, often seen in diabetic men (45), may mask the presence of PCa or delay its detection, leading to an apparent reduced risk (46), in men with impaired glucose regulation (including diabetes). Additionally, several studies have shown that serum testosterone levels are positively associated with PSA levels $(47,48)$. Thus our findings could also reflect a reduced detection of PCa in men with higher HbA1c levels, rather than any actual reduced risk of PCa development.

Strengths of our study include the large number of participants and reliable outcome data prospectively collected through linkage with national registries. Also, a comprehensive physical examination and questionnaire on measures of lifestyle was completed at cohort entry, allowing us to adjust analyses for several important confounders that have not always been available in other studies (family history, previous PSA testing).

One of the major limitations of our study was the lack of data on tumour stage and grade for $\mathrm{PCa}$, which prevented the assessment of associations between MetS components and PCa severity. Also, the relatively short follow-up (7yrs) was a limitation in relation to assessing PCa risk, especially given the cohort was quite young at enrolment (mean age 56yrs). Another limitation was that exposure variables, along with important covariates, were measured only 
once at cohort entry, so potential changes over time could not be accounted for. In addition, measures on other more specific inflammatory biomarkers were not included in the biomarker assessments undertaken in the UK Biobank cohort and anti-inflammatory medications were not taken into account in our analyses. This may have limited our findings when examining mediation via inflammatory pathways, since CRP can fluctuate in relation to various acute conditions causing inflammation $(49,50)$. Also, the "med4way" command in STATA only allowed us to analyse each mediating factor separately and not as an overall mediating effect. Finally, the homogeneous ethnic background of our cohort population ( $>94 \%$ Caucasian) may limit the generalizability of our finding to other ethnic groups.

\section{Conclusion}

Our findings suggest that there is no significant association between overall MetS and risk of PCa. In addition, HbA1c, alone or combined with other MetS components, was consistently associated with a lower risk of PCa. Our mediation analyses indicate that sex steroid hormones and inflammation may be part of the underlying biological mechanisms for this observation. However, we cannot rule out detection bias due to effects of lower testosterone on PSA levels as an explanation for lower PCa risk in men with impaired glucose regulation. Future studies should further explore the role of these potential mechanisms, especially in the context of PCa.

\section{Ethics statement}


All participants included in this analysis gave full consent for their data to be used for approved research. The UK Biobank study was approved by the North West Multi-Centre Research Ethics Committee (reference number 06/MRE08/65).

\section{Data accessibility}

The data that support the findings of this study are available from the corresponding author upon reasonable request.

\section{Conflicts of interest}

The authors have no conflicts of interest to declare

\section{Acknowledgements:}

Kerri Beckmann was supported by a National Health and Medical Research Council Early Career Researcher Fellowship (Australia) GNT1124210. 


\section{$\underline{\text { References }}$}

1. Eckel RH, Grundy SM, Zimmet PZ. The metabolic syndrome. Lancet. 2005;365(9468):1415-28.

2. Expert Panel on Detection E, Treatment of High Blood Cholesterol in A. Executive Summary of The Third Report of The National Cholesterol Education Program (NCEP) Expert Panel on Detection, Evaluation, And Treatment of High Blood Cholesterol In Adults (Adult Treatment Panel III). JAMA. 2001;285(19):2486-97.

3. Bhindi B, Locke J, Alibhai SMH, Kulkarni GS, Margel DS, Hamilton RJ, et al. Dissecting the association between metabolic syndrome and prostate cancer risk: analysis of a large clinical cohort. Eur Urol. 2015;67(1):64-70.

4. Van Hemelrijck M, Garmo H, Holmberg L, Walldius G, Jungner I, Hammar N, et al. Prostate cancer risk in the Swedish AMORIS study: the interplay among triglycerides, total cholesterol, and glucose. Cancer. 2011;117(10):2086-95.

5. Stocks T, Hergens MP, Englund A, Ye W, Stattin P. Blood pressure, body size and prostate cancer risk in the Swedish Construction Workers cohort. Int J Cancer. 2010;127(7):1660-8.

6. Stevens VL, Jacobs EJ, Maliniak ML, Patel AV, Gapstur SM. No Association of Waist Circumference and Prostate Cancer in the Cancer Prevention Study II Nutrition Cohort. Cancer Epidemiol Biomarkers Prev. 2017;26(12):1812-4.

7. Murtola TJ, Vihervuori VJ, Lahtela J, Talala K, Taari K, Tammela TL, et al. Fasting blood glucose, glycaemic control and prostate cancer risk in the Finnish Randomized Study of Screening for Prostate Cancer. Br J Cancer. 2018;118(9):1248-54.

8. Research WCRFIAIfC, Report CUP. Diet, Nutrition, Physical Activity, and Prostate Cancer 2014 [Available from: https://www.wcrf.org/sites/default/files/Prostate-Cancer-2014-Report.pdf.

9. Martin RM, Vatten L, Gunnell D, Romundstad P, Nilsen TI. Components of the metabolic syndrome and risk of prostate cancer: the HUNT 2 cohort, Norway. Cancer Causes Control. 2009;20(7):1181-92.

10. Esposito K, Chiodini P, Capuano A, Bellastella G, Maiorino MI, Parretta E, et al. Effect of metabolic syndrome and its components on prostate cancer risk: meta-analysis. J Endocrinol Invest. 2013;36(2):132-9.

11. Arthur R, Moller H, Garmo H, Holmberg L, Stattin P, Malmstrom H, et al. Association between baseline serum glucose, triglycerides and total cholesterol, and prostate cancer risk categories. Cancer Med. 2016;5(6):1307-18.

12. Papatsoris AG, Karamouzis MV, Papavassiliou AG. Novel insights into the implication of the IGF-1 network in prostate cancer. Trends Mol Med. 2005;11(2):52-5.

13. Gacci M, Russo GI, De Nunzio C, Sebastianelli A, Salvi M, Vignozzi L, et al. Meta-analysis of metabolic syndrome and prostate cancer. Prostate Cancer Prostatic Dis. 2017;20(2):146-55.

14. Hsing AW, Sakoda LC, Chua S, Jr. Obesity, metabolic syndrome, and prostate cancer. Am J Clin Nutr. 2007;86(3):s843-57.

15. Watts EL, Appleby PN, Perez-Cornago A, Bueno-de-Mesquita HB, Chan JM, Chen C, et al. Low Free Testosterone and Prostate Cancer Risk: A Collaborative Analysis of 20 Prospective Studies. Eur Urol. 2018;74(5):585-94.

16. Gao X, Bao $T$, Yang $H$, Lei $Y$, Jiang $X$, Huang $Y$, et al. The association of metabolic syndrome and its components with serum prostate-specific antigen levels. Eur J Cancer Prev. 2020;29(1):36-41.

17. Sudlow C, Gallacher J, Allen N, Beral V, Burton P, Danesh J, et al. UK biobank: an open access resource for identifying the causes of a wide range of complex diseases of middle and old age. PLoS Med. 2015;12(3):e1001779.

18. Perez-Cornago A, Key TJ, Allen NE, Fensom GK, Bradbury KE, Martin RM, et al. Prospective investigation of risk factors for prostate cancer in the UK Biobank cohort study. Br J Cancer. 2017;117(10):1562-71. 
19. Expert Panel on Detection E, Adults ToHBCi. Executive Summary of the Third Report of the National Cholesterol Education Program (NCEP) Expert Panel on Detection, Evaluation, and Treatment of High Blood Cholesterol in Adults (Adult Treatment Panel III). JAMA. 2001;285(19):2486-97.

20. Organization WH. Use of Glycated Haemoglobin (HbA1c) in the Diagnosis of Diabetes Mellitus: Abbreviated Report of a WHO Consultation. 2011.

21. Panel UBB. UK Biobank Biomarker Panel 2015 [updated 2015. 1.0:[Available from:

http://www.ukbiobank.ac.uk/wp-

content/uploads/2013/11/BCM023 ukb biomarker panel website v1.0-Aug-2015.pdf.

22. Vermeulen A, Verdonck L, Kaufman JM. A Critical Evaluation of Simple Methods for the Estimation of Free Testosterone in Serum. The Journal of Clinical Endocrinology \& Metabolism. 1999;84(10):3666-72.

23. Endert $\mathrm{E}$, Rooden $\mathrm{M}$, Fliers $\mathrm{E}$, Prummel $\mathrm{M}$, Wiersinga W. Establishment of reference values for endocrine tests-part V: acromegaly. The Netherlands journal of medicine. 2006;64:230-5.

24. Wang C, Nieschlag E, Swerdloff R, Behre HM, Hellstrom WJ, Gooren L, et al. Investigation, treatment and monitoring of late-onset hypogonadism in males: ISA, ISSAM, EAU, EAA and ASA recommendations. Eur J Endocrinol. 2008;159(5):507-14.

25. McMillan DC. Systemic inflammation, nutritional status and survival in patients with cancer. Current Opinion in Clinical Nutrition \& Metabolic Care. 2009;12(3):223-6.

26. Mackenbach JP. Health and deprivation. Inequality and the North: by P. Townsend, P. Phillimore and A. Beattie (eds.) Croom Helm Ltd, London, 1987221 pp., ISBN 0-7099-4352-0, [pound sign]8.95. Health Policy. 1988;10(2):207-6.

27. Piercy KL, Troiano RP, Ballard RM, Carlson SA, Fulton JE, Galuska DA, et al. The Physical Activity Guidelines for Americans. JAMA. 2018;320(19):2020-8.

28. Richiardi L, Bellocco R, Zugna D. Mediation analysis in epidemiology: methods, interpretation and bias. International Journal of Epidemiology. 2013;42(5):1511-9.

29. Discacciati A, Bellavia A, Lee JJ, Mazumdar M, Valeri L. Med4way: a Stata command to investigate mediating and interactive mechanisms using the four-way effect decomposition. International Journal of Epidemiology. 2018;48(1):15-20.

30. Wallner LP, Morgenstern H, McGree ME, Jacobson DJ, St Sauver JL, Jacobsen SJ, et al. The effects of metabolic conditions on prostate cancer incidence over 15 years of follow-up: results from the Olmsted County Study. BJU Int. 2011;107(6):929-35.

31. Chang SC, Yang WV. Hyperglycemia, tumorigenesis, and chronic inflammation. Crit Rev Oncol Hematol. 2016;108:146-53.

32. Dankner R, Boker LK, Boffetta P, Balicer RD, Murad H, Berlin A, et al. A historical cohort study on glycemic-control and cancer-risk among patients with diabetes. Cancer Epidemiol. 2018;57:104-9.

33. Jayedi A, Djafarian K, Rezagholizadeh F, Mirzababaei A, Hajimohammadi M, Shab-Bidar S. Fasting blood glucose and risk of prostate cancer: A systematic review and meta-analysis of dose-response. Diabetes Metab. 2018;44(4):320-7.

34. Kasper JS, Giovannucci E. A meta-analysis of diabetes mellitus and the risk of prostate cancer. Cancer Epidemiol Biomarkers Prev. 2006;15(11):2056-62.

35. Mondul AM, Weinstein SJ, Virtamo J, Albanes D. Serum total and HDL cholesterol and risk of prostate cancer. Cancer Causes Control. 2011;22(11):1545-52.

36. Van Hemelrijck M, Walldius G, Jungner I, Hammar N, Garmo H, Binda E, et al. Low levels of apolipoprotein A-I and HDL are associated with risk of prostate cancer in the Swedish AMORIS study. Cancer Causes Control. 2011;22(7):1011-9.

37. YuPeng L, YuXue Z, PengFei L, Cheng C, YaShuang Z, DaPeng L, et al. Cholesterol Levels in Blood and the Risk of Prostate Cancer: A Meta-analysis of 14 Prospective Studies. Cancer Epidemiol Biomarkers Prev. 2015;24(7):1086-93. 
38. Grundmark B, Garmo H, Loda M, Busch C, Holmberg L, Zethelius B. The metabolic syndrome and the risk of prostate cancer under competing risks of death from other causes. Cancer Epidemiol Biomarkers Prev. 2010;19(8):2088-96.

39. Rastmanesh R, Hejazi J, Marotta F, Hara N. Type 2 diabetes: a protective factor for prostate cancer? An overview of proposed mechanisms. Clin Genitourin Cancer. 2014;12(3):143-8.

40. Baradaran N, Ahmadi H, Salem S, Lotfi M, Jahani Y, Baradaran N, et al. The protective effect of diabetes mellitus against prostate cancer: role of sex hormones. Prostate. 2009;69(16):1744-50.

41. Roddam AW, Allen NE, Appleby P, Key TJ, Ferrucci L, Carter HB, et al. Insulin-like growth factors, their binding proteins, and prostate cancer risk: analysis of individual patient data from 12 prospective studies. Ann Intern Med. 2008;149(7):461-71, W83-8.

42. Renehan AG, Zwahlen M, Minder C, O'Dwyer ST, Shalet SM, Egger M. Insulin-like growth factor (IGF)-I, IGF binding protein-3, and cancer risk: systematic review and meta-regression analysis. Lancet. 2004;363(9418):1346-53.

43. Chan JM, Stampfer MJ, Giovannucci E, Gann PH, Ma J, Wilkinson P, et al. Plasma insulin-like growth factor-I and prostate cancer risk: a prospective study. Science. 1998;279(5350):563-6.

44. de Luca C, Olefsky JM. Inflammation and insulin resistance. FEBS Lett. 2008;582(1):97-105.

45. Werny DM, Saraiya M, Gregg EW. Prostate-specific antigen values in diabetic and nondiabetic US men, 2001-2002. Am J Epidemiol. 2006;164(10):978-83.

46. Beckmann K, Crawley D, Nordstrom T, Aly M, Olsson H, Lantz A, et al. Association Between Antidiabetic Medications and Prostate-Specific Antigen Levels and Biopsy Results. JAMA Netw Open. 2019;2(11):e1914689.

47. Elzanaty S, Rezanezhad B, Borgquist R. Association between PSA Levels and Biomarkers of Subclinical Systemic Inflammation in Middle-Aged Healthy Men from the General Population. Curr Urol. 2016;9(3):148-52.

48. Corona G, Boddi V, Lotti F, Gacci M, Carini M, De Vita G, et al. The relationship of testosterone to prostate-specific antigen in men with sexual dysfunction. J Sex Med. 2010;7(1 Pt 1):284-92.

49. Ugge H, Downer MK, Carlsson J, Bowden M, Davidsson S, Mucci LA, et al. Circulating inflammation markers and prostate cancer. Prostate. 2019;79(11):1338-46.

50. Sproston NR, Ashworth JJ. Role of C-Reactive Protein at Sites of Inflammation and Infection. Front Immunol. 2018;9:754. 
